ORIGINAL ARTICLE

\section{Telaprevir with Peginterferon and Ribavirin for Chronic HCV Genotype 1 Infection}

\author{
John G. McHutchison, M.D., Gregory T. Everson, M.D., Stuart C. Gordon, M.D., \\ Ira M. Jacobson, M.D., Mark Sulkowski, M.D., Robert Kauffman, M.D., \\ Lindsay McNair, M.D., John Alam, M.D., and Andrew J. Muir, M.D., \\ for the PROVEl Study Team*
}

A BSTRACT

\section{BACKGROUND}

Current therapy for chronic hepatitis $\mathrm{C}$ virus (HCV) infection is effective in less than $50 \%$ of patients infected with HCV genotype 1 . Telaprevir, a protease inhibitor specific to the HCV nonstructural 3/4A serine protease, rapidly reduced HCV RNA levels in early studies.

\section{METHODS}

We randomly assigned patients infected with HCV genotype 1 to one of three telaprevir groups or to the control group. The control group (called the PR48 group) received peginterferon alfa-2a (180 $\mu$ g per week) and ribavirin (1000 or $1200 \mathrm{mg}$ per day, according to body weight) for 48 weeks, plus telaprevir-matched placebo for the first 12 weeks ( 75 patients). The telaprevir groups received telaprevir (1250 mg on day 1 and $750 \mathrm{mg}$ every 8 hours thereafter) for 12 weeks, as well as peginterferon alfa-2a and ribavirin (at the same doses as in the PR48 group) for the same 12 weeks (the T12PR12 group, 17 patients) or for a total of 24 weeks (the T12PR24 group, 79 patients) or 48 weeks (the T12PR48 group, 79 patients). The primary outcome was a sustained virologic response (an undetectable HCV RNA level 24 weeks after the end of therapy).

\section{RESULTS}

The rate of sustained virologic response was $41 \%$ ( 31 of 75 patients) in the PR48 group, as compared with $61 \%$ (48 of 79 patients) in the T12PR24 group ( $\mathrm{P}=0.02)$, $67 \%$ (53 of 79 patients) in the T12PR48 group ( $\mathrm{P}=0.002)$, and $35 \%$ ( 6 of 17 patients) in the T12PR12 group (this group was exploratory and not compared with the control group). Viral breakthrough occurred in $7 \%$ of patients receiving telaprevir. The rate of discontinuation because of adverse events was higher in the three telaprevirbased groups ( $21 \%$, vs. $11 \%$ in the PR48 group), with rash the most common reason for discontinuation.

\section{CONCLUSIONS}

Treatment with a telaprevir-based regimen significantly improved sustained virologic response rates in patients with genotype $1 \mathrm{HCV}$, albeit with higher rates of discontinuation because of adverse events. (ClinicalTrials.gov number, NCT00336479.)
From the Duke Clinical Research Institute and Duke University, Durham, NC (J.G.M., A.J.M.); the Division of Gastroenterology and Hepatology, University of Colorado Health Science Center, Denver (G.T.E.); Division of Hepatology, Henry Ford Hospital, Detroit (S.C.G.); Division of Gastroenterology and Hepatology, Weill Cornell Medical College, New York (I.M.J.); Johns Hopkins University, Baltimore (M.S.); and Vertex Pharmaceuticals, Cambridge, MA (R.K., L.M., J.A.). Address reprint requests to Dr. McHutchison at the Duke Clinical Research Institute, Duke University Medical Center, P.O. Box 17969, Durham, NC 27715, or at mchut001@mc. duke.edu.

* Members of the Protease Inhibition for Viral Evaluation 1 (PROVEl) study team are listed in the Appendix.

N Engl J Med 2009;360:1827-38. Copyright (๑) 2009 Massachusetts Medical Society. 
W

ORLDWIDE, AN ESTIMATED 180 MILlion people have a chronic infection with hepatitis $\mathrm{C}$ virus (HCV). ${ }^{1}$ Hepatitis $\mathrm{C}$ is a leading cause of cirrhosis and hepatocellular carcinoma and is the leading indication for liver transplantation in the United States. ${ }^{2,3}$ The current treatment for HCV infection is peginterferon alfa combined with ribavirin and administered for 24 weeks (for HCV genotype 2 or 3 ) or 48 weeks (for $\mathrm{HCV}$ genotype 1 , the most prevalent genotype in Europe and North America). ${ }^{4}$ The aim of HCV therapy is a sustained virologic response, defined as an undetectable serum HCV RNA level 24 weeks after cessation of therapy. For patients with HCV genotype 1 , the rate of sustained virologic response ranges between 38 and $46 \% .^{5-7}$ In subgroups of this population, the rate of sustained virologic response is even lower, including among black patients, who have a reported rate of sustained virologic response of $19 \% .^{8}$

Efforts to improve patients' outcomes have focused on antiviral therapy specifically targeted to $\mathrm{HCV}$, and several agents in development inhibit either the HCV polymerase or protease. ${ }^{9,10}$ Telaprevir is an orally bioavailable inhibitor of the nonstructural 3/4A (NS3/4A) HCV serine protease. ${ }^{11}$ In phase $1 \mathrm{~b}$ trials, telaprevir monotherapy for 14 days resulted in a reduction of up to $5 \log _{10}$ units in plasma HCV RNA levels in subjects infected with HCV genotype $1 .{ }^{12}$ However, some subjects had evidence of viral breakthrough, and viral sequence analysis revealed resistance-associated mutations in the catalytic domain of the NS3 protease. ${ }^{13}$ Data from subsequent phase $1 \mathrm{~b}$ studies suggested that combining telaprevir with peginterferon, with or without ribavirin, increased viral inhibition and decreased the emergence of resistance. ${ }^{14-16}$

Protease Inhibition for Viral Evaluation 1 (PROVE1) was a phase $2 \mathrm{~b}$, randomized, doubleblind, multicenter study of telaprevir in combination with peginterferon alfa-2a and ribavirin in patients infected with HCV genotype 1 in the United States who had not previously been treated. We designed the study to assess the safety and efficacy of telaprevir-based therapy and to explore whether this agent could shorten the duration of the current standard therapy.

\section{METHODS}

\section{SUBJECTS}

Patients were enrolled at 37 centers in the United States. Eligible patients were 18 to 65 years of age, had a chronic genotype $1 \mathrm{HCV}$ infection, and had not been treated previously for hepatitis $\mathrm{C}$. Patients were also seronegative for hepatitis $\mathrm{B}$ surface antigen and antibodies against human immunodeficiency virus types 1 and 2, and had an absolute neutrophil count of 1500 or more per cubic millimeter, a platelet count of 90,000 or more per cubic millimeter, and a normal hemoglobin level. Patients were excluded if they had decompensated liver disease, another cause of clinically significant liver disease, hepatocellular carcinoma, or histologic evidence of cirrhosis (on liver biopsy, which was required within 2 years before the study). The protocol was approved by independent or institutional review boards of all study centers, and all patients provided written informed consent before participating in the study.

\section{STUDY DESIGN AND ORGANIZATION}

This was a phase $2 b$, randomized, parallel-group, double-blind, placebo-controlled trial. Patients who completed the screening were stratified according to self-reported race or ethnic group (black vs. any other) and baseline weight ( $>75 \mathrm{~kg}$ vs. $\leq 75 \mathrm{~kg}$ ) and were randomly assigned to one of four treatment groups. The T12PR24 group received telaprevir (VX-950, Vertex Pharmaceuticals) plus peginterferon alfa-2a (Pegasys, Roche) and ribavirin (Copegus, Roche) for 12 weeks, followed by peginterferon alfa-2a and ribavirin for 12 more weeks. The T12PR48 group received telaprevir plus peginterferon alfa-2a and ribavirin for 12 weeks, followed by peginterferon alfa-2a and ribavirin for 36 more weeks. The T12PR12 group received telaprevir plus peginterferon alfa-2a and ribavirin for 12 weeks. The PR48 (control) group received placebo plus peginterferon alfa-2a plus ribavirin for 12 weeks, followed by peginterferon alfa-2a and ribavirin for 36 more weeks.

Telaprevir was given at a dose of $1250 \mathrm{mg}$ on day 1 , followed by a dose of $750 \mathrm{mg}$ every 8 hours; peginterferon alfa-2a was given at a dose of $180 \mu \mathrm{g}$ per week by subcutaneous injection; and ribavirin 
was given orally, at a dose of $1000 \mathrm{mg}$ per day (for body weight $<75 \mathrm{~kg}$ ) or $1200 \mathrm{mg}$ per day (for body weight $\geq 75 \mathrm{~kg}$ ). Because this was the first study exploring shorter-than-standard durations of therapy ( $<48$ weeks), before initiation of the study it was planned that only 20 patients would be randomly assigned to the group with the shortest treatment period (T12PR12). For the first 80 patients, randomization was to be performed at a 1:1:1:1 ratio among the four groups (with randomization blocks of 4). The remaining 180 patients were to be randomized in a 1:1:1 ratio among the T12PR24, T12PR48, and PR48 groups (with randomization blocks of 3 ).

As is consistent with current standard practice, ${ }^{6,17}$ therapy was discontinued in patients in the PR48 group who did not have a decline of $2 \log _{10}$ units in the HCV RNA level by week 12 or undetectable HCV RNA levels at week 24. Patients in the T12PR24 and T12PR12 groups were required to have undetectable HCV RNA levels by week 4 (known as a rapid virologic response). If these patients had detectable HCV RNA at any time during weeks 4 through 20 (for T12PR24) or weeks 4 through 10 (for T12PR12), they were not permitted to undergo the assigned duration of therapy (12 or 24 weeks) and instead continued to receive peginterferon alfa-2a and ribavirin for the standard total of 48 weeks. This approach was based on the hypothesis that for patients with a slow virologic response, longer treatment with peginterferon alfa-2a would increase the likelihood of a sustained response. Because the intention was to assess the response to shorter-than-standard durations of therapy, if patients in the T12PR24 and T12PR12 groups underwent treatment for a longer period than the assigned duration, they were considered to have treatment failure in the analysis.

The study sponsor and two academic principal investigators were jointly responsible for study design and protocol development. All authors had access to the data, assume responsibility for the accuracy and completeness of the data reported, and contributed to the writing of the manuscript.

\section{EFFICACY ASSESSMENTS}

Plasma HCV RNA levels were measured with the use of the COBAS TaqMan HCV assay, version 1.0
(Roche Molecular Systems), with a lower limit of quantification of $30 \mathrm{IU}$ per milliliter and a lower limit of detection of $10 \mathrm{IU}$ per milliliter. Study visits, at which HCV RNA levels were measured, occurred at the time of screening and during the treatment period on days 1 and 4 and at weeks 1 , $2,3,4,6,8,10,12,16,20,24,28,36$, and 48 . Patients in the T12PR24 and T12PR12 groups completed visits at week 24 or 12 of the treatment period, respectively.

Patients had a safety follow-up visit 2 weeks after the completion of treatment (whether it was early or as planned). Patients who had undetectable HCV RNA levels at the time of completion of treatment had follow-up visits 4, 12, and 24 weeks afterward, at which time HCV RNA levels were measured. Patients who had detectable HCV RNA levels were not required to have further follow-up.

\section{SAFETY ASSESSMENTS}

Chemical and hematologic assessments were performed at each study visit during the treatment period and at the safety follow-up visit. At each visit, data on adverse events were collected and physical examinations were performed, if clinically indicated.

\section{VIRAL BREAKTHROUGH AND EVALUATION OF HCV SEQUENCE}

Patients were followed for viral breakthrough during the first 12 weeks of treatment. Breakthrough was defined as an increase in the HCV RNA level of $1 \log _{10}$ unit, as compared with the lowest value, or as an increase to an HCV RNA value of more than 100 IU per milliliter, if the HCV RNA had become undetectable. Patients who had viral breakthrough discontinued telaprevir or placebo but continued to receive peginterferon alfa-2a plus ribavirin for up to 48 weeks.

Blood samples were collected for viral sequencing at every study visit. Among patients who had viral breakthrough, for all samples for which the HCV RNA level was greater than 1000 IU per milliliter (the limit of detection of the sequencing assay), the NS3/4A region of the HCV genome was analyzed by means of population sequencing.

Rates of relapse during the follow-up period were calculated for the patients in each group who had undetectable HCV RNA at the time at which their assigned treatment was completed (24 weeks 
for the T12PR24 group, 12 weeks for the T12PR12 group, and 48 weeks for the T12PR48 and PR48 groups). In the T12PR 24 and T12PR12 groups, relapse rates were based on data from the patients who had a rapid virologic response at treatment week 4 and undetectable HCV RNA levels through week 20 (for T12PR24) or through week 10 (for T12PR12) and who completed therapy.

\section{STATISTICAL ANALYSIS}

The primary end point was the proportion of patients in each group who had undetectable plasma HCV RNA levels 24 weeks after the completion of therapy (sustained virologic response). Analyses of efficacy and safety included data from all patients who had undergone randomization and had received at least one dose of any study drug. The primary planned analysis, on which the sample size was based, was the comparison of the PR48 group and the T12PR 24 group. This analysis assumed a $50 \%$ response rate in the PR48 group and a $75 \%$ response rate in the T12PR 24 group, with the use of a two-sided t-test, a significance level of $5 \%$, and a sample of 80 patients who could be evaluated per group, to provide a statistical power of at least $85 \%$ to show a significant difference. The T12PR12 group was intentionally smaller than the other three groups, for an exploratory assessment of a very short duration of therapy; the study-analysis plan did not include a comparison of this group with the PR48 group.

Four planned interim analyses of safety and efficacy were conducted during the study at prespecified milestones, as was one analysis of safety only, when 80 patients had completed 12 weeks of treatment. Data management and interim analyses were performed by the Duke Clinical Research Institute; after the study was unblinded, data analyses were performed by Vertex Pharmaceuticals, the sponsor. An independent data monitoring committee reviewed the results of all interim analyses. The committee made recommendations regarding the study, but there were no predefined study-stopping rules. The primary outcome of the study was a sustained virologic response. Comparison of the primary end point between the control and telaprevir-based groups was not possible in any analysis except the final analysis, because the controls had not reached the time point at which the rate of sustained virologic response was to be calculated. Therefore, adjustments of the $\mathrm{P}$ value for the interim analyses were not necessary. All reported P values are two-sided.
Figure 1 (facing page). Screening, Randomization, and Sustained Virologic Response (SVR) among the Study Patients.

For the 66 patients who did not meet inclusion criteria, the reasons were as follows: not meeting the entry criteria (47 patients), withdrawal of consent (11), being enrolled at a site that withdrew from the study (5), failure to complete screening visits (2), and having an injury before day 1 that prevented study dosing (1). Randomization was performed 5 to 7 days before the start of dosing to allow for time for shipment of the study drug to the site. Of the 13 patients who withdrew after randomization, 11 declined to participate, 1 attempted to participate but withdrew consent when venous access could not be obtained for the predosing blood drawing on day 1 , and 1 could not be given a study drug because documentation of eligibility was still pending when the period of enrollment closed. The 11 patients in the T12PR24 group and the 4 in the T12PR12 group who continued to receive peginterferon alfa-2a and ribavirin did so because of a lack of protocol-defined virologic response, so even if they later had a sustained virologic response (defined as an undetectable HCV RNA level 24 weeks after completion of study treatment), they were not counted among the total number of patients with a sustained virologic response. The protocol-defined virologic stopping rule, met by 20 patients, was applicable only to the PR48 (control) group.

\section{RESULTS}

\section{CHARACTERISTICS OF THE PATIENTS}

Between June 2006 and September 2006, a total of 263 patients underwent randomization, and 250 received at least one dose of a study drug (Fig. 1). The baseline characteristics were similar among the treatment groups (Table 1). The majority of patients (157 of 250 [63\%]) were men, and the mean age was 48.1 years. Whites accounted for $77 \%$ of the study population (192 of $250 \mathrm{pa}-$ tients). Most patients (218 of 250 [87\%]) had HCV RNA levels of 800,000 IU or more per milliliter at baseline.

\section{EFFICACY}

The sustained virologic response rate was $61 \%$ (48 of 79 patients) in the T12PR24 group, as compared with $41 \%$ (31 of 75 patients) in the PR48 group ( $\mathrm{P}=0.02)$ (Table 2). Rates of sustained virologic response were $67 \%$ (53 of 79 patients) in the T12PR48 group $(\mathrm{P}=0.002$ and $\mathrm{P}=0.51$ for the comparison with the PR48 group and the T12PR24 group, respectively) and 35\% (6 of 17 patients) in the T12PR12 group (Table 2). In the small subgroup of black patients enrolled in the study, rates of sustained virologic response were $11 \%$ ( 1 of 9 


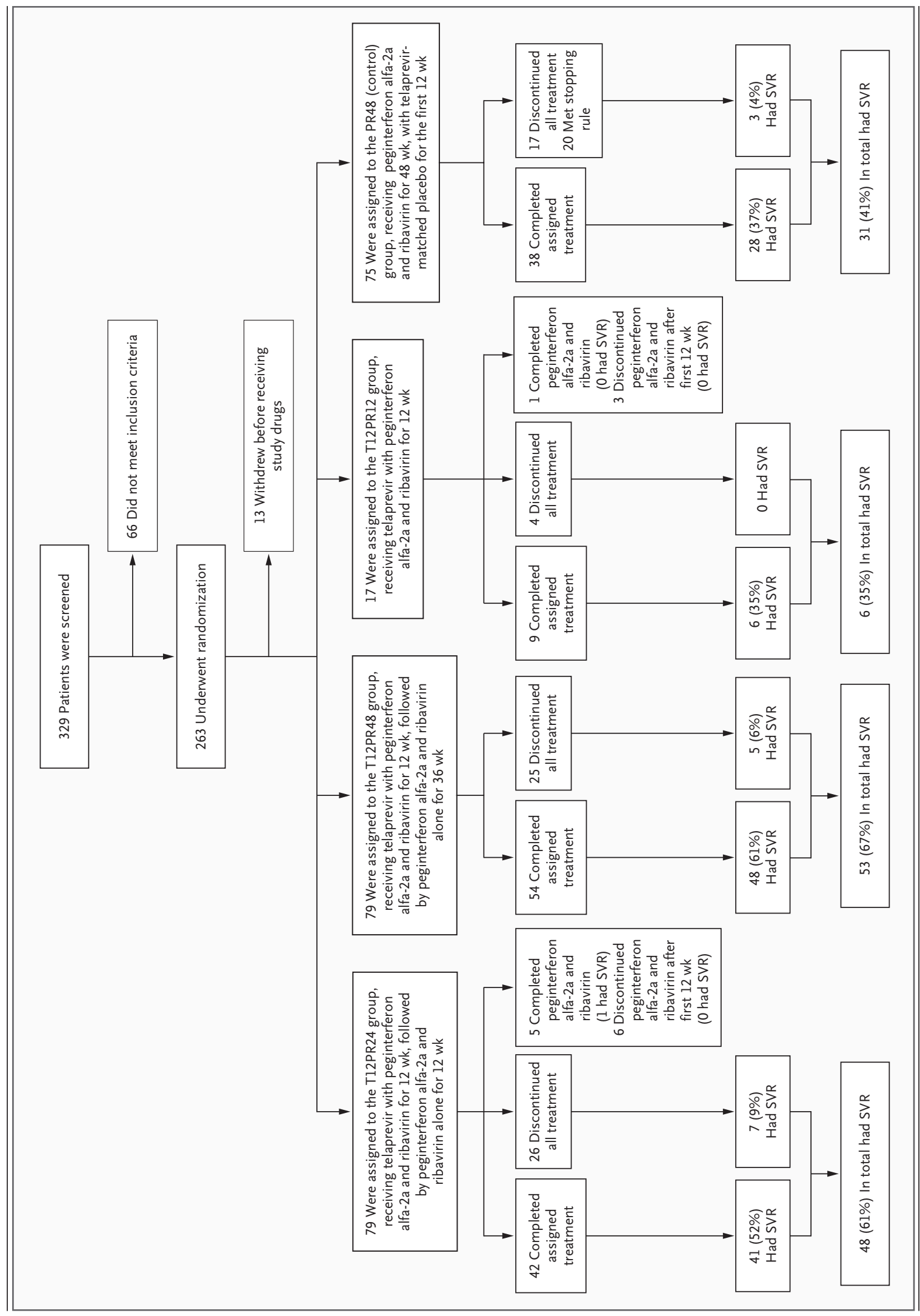




\begin{tabular}{|c|c|c|c|c|}
\hline Characteristic & T12PR24 (N=79) & T12PR48 ( $N=79)$ & T12PR12 ( $=17)$ & PR48 $(\mathrm{N}=75)$ \\
\hline Years of age - median (range) & $49(21-61)$ & $50(26-61)$ & $49(34-63)$ & $49(24-59)$ \\
\hline $\mathrm{BMI}$ - median (range) $\dagger$ & $26.9(18-41)$ & $25.8(19-44)$ & $28.6(20-38)$ & $26.9(19-38)$ \\
\hline Male sex — no. (\%) & $54(68)$ & $48(61)$ & $12(71)$ & $43(57)$ \\
\hline \multicolumn{5}{|l|}{ Race or ethnic group — no. (\%) } \\
\hline White & $60(76)$ & $60(76)$ & $13(76)$ & $59(79)$ \\
\hline Black & $7(9)$ & $8(10)$ & $3(18)$ & $9(12)$ \\
\hline Asian & $1(1)$ & $3(4)$ & 0 & 0 \\
\hline Hispanic & $9(11)$ & $7(9)$ & $1(6)$ & $6(8)$ \\
\hline Other & $2(3)$ & $1(1)$ & 0 & $1(1)$ \\
\hline \multicolumn{5}{|l|}{ HCV genotype 1 subtype - no. (\%) } \\
\hline la & $53(67)$ & $48(61)$ & $9(53)$ & $50(67)$ \\
\hline $1 b$ & $17(22)$ & $27(34)$ & $6(35)$ & $20(27)$ \\
\hline Indeterminate & $9(11)$ & $4(5)$ & $2(12)$ & $5(7)$ \\
\hline HCV RNA - $\log _{10} I U / \mathrm{ml} \sqrt{ }$ & $6.54 \pm 0.72$ & $6.47 \pm 0.60$ & $6.57 \pm 0.43$ & $6.68 \pm 0.49$ \\
\hline HCV RNA $\geq 800,000 \mathrm{IU} / \mathrm{ml}$ - no. (\%)』 & $66(84)$ & $68(86)$ & $15(88)$ & $69(92)$ \\
\hline \multicolumn{5}{|l|}{ Fibrosis - no. (\%) } \\
\hline None or minimal & $24(30)$ & $34(43)$ & $4(24)$ & $19(25)$ \\
\hline Portal & $41(52)$ & $31(39)$ & $9(53)$ & $37(49)$ \\
\hline Bridging & $14(18)$ & $14(18)$ & $4(24)$ & $19(25)$ \\
\hline $\mathrm{ALT}-\mathrm{IU} / \mathrm{ml}$ & $73 \pm 54$ & $72 \pm 49$ & $80 \pm 75$ & $68 \pm 38$ \\
\hline Total bilirubin $-\mu \mathrm{mol} /$ liter & $10.5 \pm 3.3$ & $10.8 \pm 4.6$ & $11.4 \pm 4.6$ & $9.9 \pm 3.7$ \\
\hline INR for prothrombin time & $1.02 \pm 0.08$ & $1.01 \pm 0.10$ & $1.02 \pm 0.09$ & $1.03 \pm 0.11$ \\
\hline Serum albumin $-\mathrm{g} /$ liter & $41.6 \pm 2.8$ & $41.7 \pm 2.5$ & $41.2 \pm 2.2$ & $40.7 \pm 3.1$ \\
\hline Platelet count $-\times 10^{-9} /$ liter & $225.9 \pm 58.4$ & $223.8 \pm 64.9$ & $213.9 \pm 56.8$ & $231.1 \pm 60.9$ \\
\hline
\end{tabular}

* Plus-minus values are means \pm SD. Patients were randomly assigned to one of three telaprevir groups or to a control group. The control group (called the PR48 group) received peginterferon alfa-2a (180 $\mathrm{kg}$ per week) and ribavirin (1000 or $1200 \mathrm{mg}$ per day, according to body weight) for 48 weeks, plus telaprevir-matched placebo for the first 12 weeks. The telaprevir groups received telaprevir ( $1250 \mathrm{mg}$ on day 1 and $750 \mathrm{mg}$ every 8 hours thereafter) for 12 weeks, as well as peginterferon alfa-2a and ribavirin (at the same doses as in the PR48 group) for the same 12 weeks (the T12PR12 group) or for a total of 24 weeks (the T12PR24 group) or 48 weeks (the T12PR48 group). Baseline factors were compared among the PR48 group, the T12PR24 group, and the T12PR48 group. None of the listed characteristics were significantly different, except for the baseline hepatitis C virus (HCV) RNA levels in the PR48 and T12PR48 groups

$(P=0.03)$, but the difference was not clinically meaningful. To convert values for total bilirubin to milligrams per deciliter, divide by 17.1. ALT denotes alanine aminotransferase, and INR international normalized ratio.

$\uparrow$ The body-mass index (BMI) is the weight in kilograms divided by the square of the height in meters.

$\checkmark$ Race or ethnic group was self-reported.

$\int$ The HCV RNA level was measured with the use of the COBAS TaqMan assay (Roche), which has a lower limit of quantification of $30 \mathrm{IU}$ per milliliter.

patients) in the PR48 group and 44\% (8 of 18 patients) in the telaprevir-based groups.

Rates of rapid virologic response (undetectable HCV RNA levels at week 4) were higher with telaprevir-based therapy than without it (in the PR48 group) ( $\mathrm{P}<0.001$ for each comparison) (Table 2 and Fig. 2). In the T12PR24 and T12PR12 groups, patients who had neither a rapid virologic response nor HCV RNA levels that remained un- detectable through week 20 (for T12PR24) or through week 10 (for T12PR12), were to continue treatment with peginterferon and ribavirin for 48 weeks. Of the 15 patients who were to continue treatment, 9 discontinued therapy before it was completed and 1, in the T12PR24 group, completed the 48 weeks of treatment and had a sustained virologic response (Fig. 1). In the analyses, this patient was counted as not having had a response. 


\begin{tabular}{|c|c|c|c|c|}
\hline \multirow[t]{3}{*}{ Week } & \multicolumn{4}{|c|}{ Undetectable Viral RNA } \\
\hline & T12PR24 (N=79) & T12PR48 (N=79) & T12PR12 (N=17) & PR48 $(N=75)$ \\
\hline & \multicolumn{4}{|c|}{ number (percent) } \\
\hline \multicolumn{5}{|l|}{ Treatment week } \\
\hline 4 & $64(81)$ & $64(81)$ & $10(59)$ & $8(11)$ \\
\hline 12 & $54(68)$ & $63(80)$ & $12(71)$ & $34(45)$ \\
\hline 24 & $45(57)$ & $56(71)$ & NA & $43(57)$ \\
\hline 48 & NA & $51(65)$ & NA & $35(47)$ \\
\hline $\begin{array}{l}\text { Follow-up week } 24 \text {, when SVR was } \\
\text { assessed } †\end{array}$ & $48(61)$ & $53(67)$ & $6(35)$ & $31(41)$ \\
\hline \multicolumn{5}{|c|}{$\begin{array}{l}\text { Undetectable was defined as less than } 10 \text { IU per milliliter, the limit of detection of the assay used (COBAS TaqMan as } \\
\text { say, Roche). Sustained virologic response (SVR) was defined as an undetectable HCV RNA level } 24 \text { weeks after comple } \\
\text { tion of the study drugs in patients who completed the assigned treatment or who discontinued treatment early. The } \\
\text { PR48 group was the control group. NA denotes not applicable. } \\
\text { TAt follow-up week } 24 \text {, when the SVR status was ascertained, the proportion of patients with undetectable HCV RNA } \\
\text { levels in the T12PR24 group was significantly greater than that in the PR48 group }(P=0.02) \text { but not than that in the } \\
\text { T12PR48 group }(P=0.51) \text {, and the proportion in the T12PR48 group was significantly greater than that in the PR48 } \\
\text { group }(P=0.002) \text {. }\end{array}$} \\
\hline
\end{tabular}

At baseline, $20 \%$ of the patients in the PR48 group and $25 \%$ of the patients in the telaprevirbased groups had alanine aminotransferase values within the normal range. At the end of the treatment period, $75 \%$ of patients in the PR48 group and $76 \%$ of those in the telaprevir-based groups had normal alanine aminotransferase values.

Only 1 of 41 patients (2\%) in the T12PR 24 group had a relapse (undetectable HCV RNA at the time of completion of treatment but detectable levels during the follow-up period), whereas 3 of 51 patients $(6 \%)$ in the T12PR48 group and 3 of 9 patients (33\%) in the T12PR12 group had a relapse. In the PR48 group, 8 of 35 patients (23\%) had a relapse.

Among the telaprevir-treated patients, 7\% (12 of 175) had viral breakthrough (an increase of $>1 \log _{10}$ unit of HCV RNA as compared with the lowest value during the treatment period, or if the HCV RNA level had become undetectable, an increase to an HCV RNA value of $>100$ IU per milliliter). Most breakthroughs occurred during weeks 1 through 4 (in 9 of 12 patients), with the remainder (in 3 patients) occurring between weeks 5 and 12. Ten of the 12 patients had breakthroughs before the HCV RNA level became undetectable. The viral population at the time of breakthrough consisted predominantly of variants containing mutations V36M and R155K (in 10 patients infected with HCV genotype 1a) or A156T (in 1 patient with
HCV genotype 1b). The remaining patient had missed several days of dosing and had a breakthrough at day 8; this patient was infected with predominantly wild-type virus.

\section{SAFETY}

The most common adverse events were consistent with typical interferon-induced systemic symptoms (Table 3). However, certain adverse events - such as rash, pruritus, nausea, and diarrhea - were more common in the groups that received telaprevir. The proportion of patients who discontinued treatment because of an adverse event was higher in the three telaprevir-based treatment groups (21\%) than in the PR48 group (11\%) (Table 4). Serious adverse events were reported in 22 patients during treatment (4 patients in the PR48 group and 18 patients in the telaprevir-based groups). Fifteen of the serious adverse events were considered to be related to the study drug regimen. Serious adverse events in more than one patient, which occurred only in the telaprevir-based groups, included rash (three patients), anemia (three patients), ocular events (retinal detachment and scotoma, three patients), and depression (two patients).

Rash was more common in the telaprevir-based groups than in the control group, with mild rash occurring in $37 \%$ and $32 \%$ of patients, respectively; moderate rash, in $15 \%$ and $8 \%$, respectively; and severe rash, in $7 \%$ and $1 \%$, respectively. The rashes 


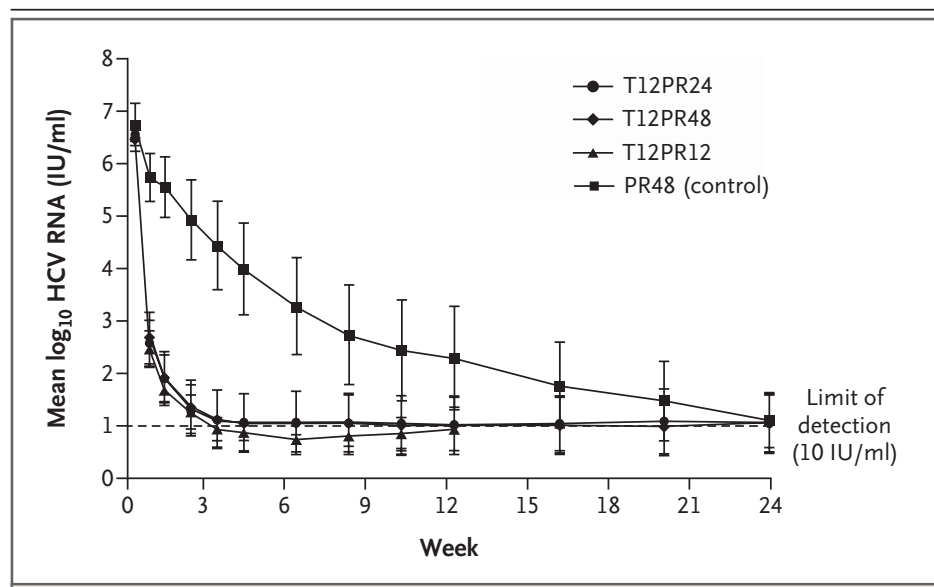

Figure 2. Mean $\log _{10}$ HCV RNA Levels from Baseline through Week 24, According to Treatment Group.

Data are shown only through treatment week 24 because all patients who continued the assigned treatment beyond that time had undetectable HCV RNA. Data are shown for 79 patients in the T12PR24 group, 79 in the T12PR48 group, 17 in the T12PR12 group, and 75 in the PR48 (control) group. The I bars indicate standard deviations.

were described as typical drug-induced maculopapular rashes. Pruritus was sometimes, but not always, considered to be associated with rash. The site investigators determined the severity of the rashes. When it became clear that there was an increased incidence of rash, the protocol was amended to include guidance for the grading of rash, but few rashes occurred after that time. The protocol did not provide specific guidance for discontinuation of treatment because of rash, although in most cases of severe rash, one or more of the study drugs was stopped. The median time to treatment discontinuation because of rash in the telaprevir groups was 73 days (range, 8 to 88 ) after the start of the study treatment. All cases of severe rash, which were followed by the site investigators and medical monitors, resolved after the discontinuation of treatment.

Changes in laboratory values during the study were consistent with those reported in association with the combined use of peginterferon and ribavirin. ${ }^{6,18}$ A decrease in hemoglobin levels was more common in patients receiving telaprevirbased regimens than in controls during the first 12 weeks of treatment. Erythropoietin use was prohibited during the first 12 weeks of treatment to allow for accurate assessment of any potential effect on hemoglobin levels. Patients in the PR48 group had a median decline in the hemoglobin level of about $3 \mathrm{~g}$ per deciliter at week 12; the decline in the telaprevir groups was 0.5 to $1 \mathrm{~g}$ per deciliter greater. After telaprevir was discontinued, the hemoglobin values increased to the mean level in the control group and remained similar to control levels thereafter. No other clinically significant differences in laboratory abnormalities were noted between the telaprevir groups and the control group.

\section{DISCUSSION}

The results of this study, as well as those of a similar study conducted in Europe (the PROVE2 study, reported elsewhere in this issue of the Journal), ${ }^{19}$ suggest that the addition of a hepatitis Cspecific protease inhibitor, telaprevir, to current standard therapy consisting of peginterferon alfa$2 \mathrm{a}$ and ribavirin can significantly improve the rate of sustained virologic response in patients infected with HCV genotype 1 , as compared with the rate with standard therapy. The observed sustained virologic response in the PR48 group (41\%) is consistent with previous reports..$^{5-7}$ Our study also shows that the duration of therapy can be reduced from 48 weeks to 24 weeks for most patients while maintaining an improved sustained virologic response. The overall responses reflect a marked increase in the rate of rapid virologic response at week 4, and a low subsequent rate of relapse, with telaprevir-based treatment as compared with standard therapy. Although there were relatively few black patients treated in this study, the rate of sustained virologic response of $44 \%$ among black patients in the telaprevir-based groups is encouraging.

The relapse rates that we observed for the 24week and 48-week treatment regimens that included telaprevir ( $2 \%$ and $6 \%$, respectively) were lower than the relapse rate observed in the control group (23\%); the rate in the control group was within the range of the reported rates of 18 to $30 \%$ for current standard HCV antiviral therapy. 5,20 Moreover, the relapse rate of $2 \%$ in the T12PR 24 group indicates that a 24 -week treatment duration is sufficient in patients who have a rapid virologic response. Patients randomly assigned to the T12PR24 group who did not have a rapid virologic response received peginterferon and ribavirin for an extended period - 48 weeks. Therefore, this study cannot evaluate whether 24-week treatment would be sufficient in this subgroup. 


\begin{tabular}{|c|c|c|c|c|}
\hline \multirow[t]{2}{*}{ Adverse Event } & T12PR24 (N=79) & T12PR48 ( $N=79)$ & T12PR12 ( $\mathrm{N}=17)$ & PR48 ( $N=75)$ \\
\hline & \multicolumn{4}{|c|}{ percent of patients } \\
\hline Fatigue & 70 & 73 & 82 & 76 \\
\hline Nausea & 56 & 48 & 65 & 29 \\
\hline Influenza-like illness & 49 & 38 & 35 & 43 \\
\hline Pruritus & 48 & 40 & 24 & 23 \\
\hline Headache & 47 & 43 & 53 & 60 \\
\hline Insomnia & 44 & 34 & 35 & 39 \\
\hline Diarrhea & 42 & 34 & 24 & 28 \\
\hline Anemia & 37 & 29 & 35 & 27 \\
\hline \multicolumn{5}{|l|}{ Rash'̈ } \\
\hline Any & 60 & 61 & 53 & 41 \\
\hline Severe & 9 & 5 & 6 & 1 \\
\hline Moderate & 18 & 17 & 0 & 8 \\
\hline Mild & 33 & 39 & 47 & 32 \\
\hline Erythema at injection site & 28 & 32 & 35 & 24 \\
\hline Dizziness & 28 & 19 & 24 & 19 \\
\hline Vomiting & 24 & 20 & 18 & 12 \\
\hline Depression & 22 & 19 & 12 & 17 \\
\hline Cough & 22 & 20 & 18 & 19 \\
\hline Pyrexia & 20 & 19 & 12 & 29 \\
\hline Dry skin & 18 & 17 & 6 & 25 \\
\hline Irritability & 17 & 10 & 12 & 29 \\
\hline Arthralgia & 17 & 22 & 24 & 21 \\
\hline Dyspnea & 15 & 13 & 18 & 15 \\
\hline Neutropenia & 14 & 24 & 0 & 24 \\
\hline Hemorrhoids & 13 & 17 & 24 & 1 \\
\hline Blurred vision & 13 & 17 & 17 & 8 \\
\hline Pain & 11 & 8 & 6 & 20 \\
\hline Myalgia & 11 & 19 & 18 & 24 \\
\hline Chills & 10 & 23 & 18 & 19 \\
\hline Generalized pruritus & 10 & 18 & 18 & 0 \\
\hline Alopecia & 10 & 17 & 0 & 11 \\
\hline Anxiety & 8 & 14 & 18 & 17 \\
\hline Back pain & 8 & 10 & 12 & 16 \\
\hline Disturbance in attention & 5 & 8 & 18 & 9 \\
\hline Dyspnea on exertion & 5 & 6 & 18 & 11 \\
\hline
\end{tabular}

* The adverse events listed are those that were reported in at least $15 \%$ of patients in any of the four study groups; all grades of events were included. Events in bold are those in which one or more of the three groups receiving telaprevir had an event incidence that was 10 percentage points higher than that in the PR48 (control) group.

$\dagger$ Determination of the severity of rashes was performed by the site investigators. The protocol was amended to include guidance for the grading of rash when the increased incidence of rash was recognized, but most patients had completed the first 12 weeks of the study by the time of the amendment, and few rashes occurred after that time. 


\begin{tabular}{|c|c|c|c|c|}
\hline \multirow[t]{3}{*}{ Adverse Event } & \multicolumn{2}{|c|}{ All Telaprevir-Based Regimens $(\mathrm{N}=175)$} & \multicolumn{2}{|c|}{ PR48 (N=75) } \\
\hline & Weeks $1-12 *$ & After Week 12 & Weeks $1-12 *$ & After Week 12 \\
\hline & \multicolumn{4}{|c|}{ number of patients (percent) } \\
\hline Any & $31(18)$ & $6(3)$ & $3(4)$ & $5(7)$ \\
\hline Rash or pruritus & $12(7)$ & 0 & 0 & $1(1)$ \\
\hline Anemia & $3(2)$ & 0 & 0 & 0 \\
\hline Gastrointestinal event & $2(1)$ & $2(1)$ & $1(1)$ & 0 \\
\hline Psychiatric event (depression or anxiety) & $4(2)$ & $1(1)$ & $1(1)$ & 0 \\
\hline Other event $†$ & $7(4)$ & $2(1)$ & $1(1)$ & $4(5)$ \\
\hline Multiple events: & $3(2)$ & $1(1)$ & 0 & 0 \\
\hline
\end{tabular}

* Weeks 1 through 12 were when patients received telaprevir or matching placebo. The PR48 group was the control group.

$\uparrow$ Other events in the PR48 group were arthralgia, pneumonia, eye pain, relapse of narcotics use, and dyspnea on exertion. Other events in the three telaprevir-based groups during weeks 1 through 12 were eye scotoma, cellulitis, dizziness, myocardial infarction, thrombocytopenia, folliculitis on legs, and esophageal candidiasis. Other events in the telaprevir-based groups after week 12 were furuncle and cyst over eye.

$¥$ Multiple events consisted of several simultaneous adverse events, with action taken for each reported as discontinuation of treatment. The three patients with multiple events during weeks 1 through 12 were one patient with fatigue, insomnia, and loss of appetite; one with vomiting, dehydration, and increased blood urea nitrogen, creatinine, and uric acid levels; and one with muscle cramps, weight loss, headache, itching, and influenza-like symptoms. After week 12, one patient had multiple events: cough, anemia, hair loss, tooth pain, and thyroid dysfunction.

The addition of telaprevir to therapy with peginterferon alfa-2a and ribavirin was associated with an increase in the rate of treatment discontinuation, predominantly owing to the side effect of rash. In a previous study of telaprevir and peginterferon alfa-2a, rash was more common in the two groups receiving telaprevir than in the group receiving peginterferon alfa-2a alone. ${ }^{15}$ Guidelines for the management of rash have been incorporated in all subsequent clinical protocols involving telaprevir; the guidelines were added to this protocol, but almost all patients had completed the telaprevir-dosing period by that time, so the effect on the outcome in this study cannot be assessed. The exact mechanism of action leading to rash in patients receiving telaprevir remains unknown. An adverse effect on hemoglobin was also noted with telaprevir-based regimens; this effect was of low magnitude and was reversed within 4 weeks after telaprevir dosing ended.

This study was designed to evaluate telaprevir in combination with peginterferon alfa-2a and ribavirin in a treatment regimen with regard to the rate of sustained virologic response and to compare telaprevir-based treatment with current therapy. With the addition of peginterferon alfa-2a and ribavirin to the telaprevir-based regimen, vi- ral breakthrough was infrequent (seen in $7 \%$ of patients). Viral variants observed in patients at the time of breakthrough in this study carried telaprevir-resistance-associated mutations similar to those described in studies of shorter duration. ${ }^{16}$ These variants are sensitive to interferon and ribavirin in vitro ${ }^{21}$ and have been shown previously to respond to subsequent interferon and ribavirin treatment in some patients. ${ }^{16}$ Theoretically, the patients with viral breakthrough may have had a reduced response to peginterferon and ribavirin. ${ }^{13,16}$ The potential long-term clinical consequences of the selection and persistence of these variants in patients who do not achieve a sustained virologic response are unknown and will require long-term follow-up studies.

In conclusion, the addition of telaprevir to peginterferon alfa-2a and ribavirin in patients infected with hepatitis $\mathrm{C}$ genotype 1 who had not been treated previously significantly increased the rate of sustained virologic response; this approach may allow for a substantial reduction in the duration of therapy in most patients.

Supported by Vertex Pharmaceuticals.

Dr. McHutchison reports receiving consulting fees from Vertex and Schering-Plough and grant support from Schering-Plough, Roche, and Vertex; Dr. Everson, consulting fees from Vertex and Roche, lecture fees from Roche, and grant support from Vertex 
and Roche; Dr. Gordon, consulting fees from Vertex, lecture fees from Schering-Plough and Roche, and grant support from GlaxoSmithKline, Valeant, Schering-Plough Research Institute, Gilead, GlobeImmune, Conatus, Merck, Roche, Vertex, Human Genome Sciences, Coley, Phynova, Exalenz, Echosens, Biolex, Bristol-Myers Squibb, Idera, Intercept, and SciClone; Dr. Jacobson, consulting fees and grant support from Vertex; and Dr. Sulkowski, consulting fees from Vertex, Schering-Plough, Roche, Merck, Human Genome Sciences, and Boehringer Ingelheim, lecture fees from Schering-Plough and Roche, and grant support from Vertex. Dr. Kauffman reports owning equity in Vertex. Drs. Kauffman, McNair, and Alam report being current or former employees of Vertex Pharmaceuticals and holding stock options in this entity. Dr. Muir reports receiving consulting fees from Vertex and ScheringPlough, lecture fees from Schering-Plough, and grant support from Vertex and Schering-Plough. No other potential conflict of interest relevant to this article was reported.

We thank the study coordinators, nurses, and patients involved in the study; Shelley George and Nathalie Adda for their medical oversight, Jennifer Webster for clinical project management, Leif Bengtsson for statistical analyses, and Tara Kieffer for viral sequencing work (all five from Vertex Pharmaceuticals); Melissa Banks and Casey Hale, for clinical project management (both from Duke Clinical Research Institute [DCRI], the coordinating center for the study); and Jennifer King (August Editorial, Raleigh, NC, with funding from DCRI) and Paula Michelle del Rosario (Gardiner-Caldwell Communications, Macclesfield, United Kingdom, with funding from Vertex Pharmaceuticals) for editorial assistance in collating author contributions and manuscript formatting before submission.

\section{APPENDIX}

The members of the PROVE1 study team included the following: N. Afdhal, Beth Israel Deaconess Medical Center Liver Center, Boston; S. Arora, University of New Mexico School of Medicine, Albuquerque; V. Balan and H. Vargas, Mayo Clinic Hospital, Phoenix, AZ; D. Bernstein, North Shore University Hospital, Manhasset, NY; M. Black, Temple University Hospital, Philadelphia; R. Brown, Columbia University Medical Center, New York; N. Bzowej, California Pacific Medical Center, San Francisco; G. Davis, Baylor University Medical Center, Dallas; A. Di Bisceglie, Saint Louis University, St. Louis; J. Dienstag, Massachusetts General Hospital, Boston; G. Everson, University of Colorado, Aurora; S. Faruqui, Gulf Coast Research Association, Baton Rouge, LA; J. Franco, Medical College of Wisconsin, Milwaukee; M. Fried, University of North Carolina Hospitals, Chapel Hill; R. Ghalib, Liver Institute at Methodist Dallas, Dallas; S.C. Gordon, Henry Ford Hospital, Detroit; J. Gross, Mayo Clinic, Rochester, MN; I.M. Jacobson, Weill Cornell Medical College, New York; D. Jensen, University of Chicago, Chicago; M. Kugelmas, South Denver Gastroenterology, Englewood, CO; P. Kwo, Indiana University School of Medicine, Indianapolis; E. Lawitz, Alamo Medical Research, San Antonio, TX; W. Lee, University of Texas Southwestern Medical Center, Dallas; P. Martin, Mount Sinai Hospital, New York; D. Nelson, University of Florida Gainesville, Gainesville; P. Northup, University of Virginia Health Systems, Charlottesville; K. Patel, Duke University Medical Center, Durham, NC; F. Poordad, CedarsSinai Medical Center, Los Angeles; R.K. Reddy, University of Pennsylvania Hospital, Philadelphia; M. Rodriguez-Torres, Fundacion de Investigation de Diego, Santurce, Puerto Rico; V. Rustgi, Metropolitan Research, Fairfax, VA; E. Schiff, University of Miami, Miami; K. Sherman, University of Cincinnati Medical Center, Cincinnati; M. Shiffman, Virginia Commonwealth University Medical Center, Richmond; M. Sulkowski, Johns Hopkins University School of Medicine, Baltimore; G. Szabol, University of Massachusetts Memorial Medical Center, Worcester; Z. Younossi, Inova Fairfax Hospital, Annandale, VA.

\section{REFERENCES}

1. World Health Organization Initiative for Vaccine Research. Hepatitis C. (Accessed April 6, 2009, at http://www.who. int/vaccine_research/diseases/viral_ cancers/en/index2.html.)

2. Di Bisceglie AM, Lyra AC, Schwartz $\mathrm{M}$, et al. Hepatitis C-related hepatocellular carcinoma in the United States: influence of ethnic status. Am J Gastroenterol 2003; 98:2060-3.

3. Organ Procurement and Transplantation Network. U.S. liver transplants performed: January 1, 1988-April 30, 2008. (Accessed April 6, 2009, at http://www. optn.org/.)

4. World Health Organization. Global surveillance and control of hepatitis C: report of a WHO consultation organized in collaboration with the Viral Hepatitis Prevention Board, Antwerp, Belgium. J Viral Hepat 1999;6:35-47.

5. Manns MP, McHutchison JG, Gordon SC, et al. Peginterferon alfa-2b plus ribavirin compared with interferon alfa-2b plus ribavirin for initial treatment of chronic hepatitis C: a randomised trial. Lancet 2001; 358:958-65.

6. Fried MW, Shiffman ML, Reddy KR, et al. Peginterferon alfa-2a plus ribavirin for chronic hepatitis $\mathrm{C}$ virus infection. N Engl J Med 2002;347:975-82.

7. Sulkowski M, Lawitz E, Shiffman ML, et al. Final results of the phase 3b IDEAL (individualized dosing efficacy versus flat dosing to assess optimal pegylated interferon therapy) phase IIIb study. Presented at the 43rd Annual Meeting of the European Association for the Study of the Liver (EASL), Milan, April 23-27, 2008. abstract. 8. Muir AJ, Bornstein JD, Killenberg PG. Peginterferon alfa-2b and ribavirin for the treatment of chronic hepatitis $\mathrm{C}$ in blacks and non-Hispanic whites. N Engl J Med 2004;350:2265-71. [Erratum, N Engl J Med 2004;351:1268.]

9. Pawlotsky JM, Chevaliez S, McHutchison JG. The hepatitis $\mathrm{C}$ virus life cycle as a target for new antiviral therapies. Gastroenterology 2007;132:1979-98.

10. Manns MP, Foster GR, Rockstroh JK, Zeuzem S, Zoulim F, Houghton M. The way forward in HCV treatment - finding the right path. Nat Rev Drug Discov 2007;6:991-1000. [Errata, Nat Rev Drug Discov 2008;7:102, 458.]

11. Lin C, Kwong AD, Perni RB. Discovery and development of VX-950, a novel, covalent, and reversible inhibitor of hepatitis C virus NS3.4A serine protease. Infect Disord Drug Targets 2006;6:3-16.

12. Reesink HW, Zeuzem S, Weegink CJ, et al. Rapid decline of viral RNA in hepatitis C patients treated with VX-950: a phase Ib, placebo-controlled, randomized study. Gastroenterology 2006;131:997-1002.

13. Sarrazin C, Kieffer TL, Bartels D, et al. Dynamic hepatitis C virus genotypic and phenotypic changes in patients treated with the protease inhibitor telaprevir. Gastroenterology 2007;132:1767-77.

14. Lawitz E, Rodriguez-Torres M, Muir $\mathrm{AJ}$, et al. Antiviral effects and safety of telaprevir, peginterferon alfa-2a, and ribavirin for 28 days in hepatitis $\mathrm{C}$ patients. J Hepatol 2008;49:163-9.

15. Forestier N, Reesink HW, Weegink CJ, et al. Antiviral activity of telaprevir (VX-950) and peginterferon alfa-2a in patients with hepatitis C. Hepatology 2007;46:640-8.

16. Kieffer TL, Sarrazin C, Miller JS, et al. Telaprevir and pegylated interferon-alpha$2 \mathrm{a}$ inhibit wild-type and resistant genotype 1 hepatitis $\mathrm{C}$ virus replication in patients. Hepatology 2007;46:631-9.

17. Dienstag JL, McHutchison JG. American Gastroenterological Association technical review on the management of 
hepatitis C. Gastroenterology 2006;130: 231-64.

18. Hadziyannis SJ, Sette H Jr, Morgan TR, et al. Peginterferon-alpha2a and ribavirin combination therapy in chronic hepatitis C: a randomized study of treatment duration and ribavirin dose. Ann Intern Med 2004;140:346-55.

19. Hézode C, Forestier N, Dusheiko G, et al. Telaprevir and peginterferon with or without ribavirin for chronic $\mathrm{HCV}$ infection. N Engl J Med 2009;360:1839-50.

20. Jacobson IM, Brown RS Jr, Freilich B, et al. Peginterferon alfa-2b and weightbased or flat-dose ribavirin in chronic hepatitis C patients: a randomized trial. Hepatology 2007;46:971-81.

21. Zhou Y, Bartels D, Muh U, et al. Phe- notypic characterization of telaprevir (VX950) NS3 protease variants in a HCV replicon system. Presented at the 13th International Symposium on Hepatitis C Virus and Related Viruses, Cairns, Australia, August 27-31, 2006.

Copyright $(2009$ Massachusetts Medical Society. 